\title{
Robot Path Generation Method for a Welding System Based on Pseudo Stereo Visual Servo Control
}

\author{
Theodore P. Pachidis \\ Department of Electrical and Computer Engineering, Democritus University of Thrace, Xanthi 67100, Greece \\ Email:pated@otenet.gr
}

Kostas N. Tarchanidis

Department of Petroleum Technology, Technological Educational Institute of Kavala, Ag Loukas, Kavala 65404, Greece Email: ktarch@teikav.edu.gr

John N. Lygouras

Department of Electrical and Computer Engineering, Democritus University of Thrace, Xanthi 67100, Greece Email: ilygour@ee.duth.gr

Philippos G. Tsalides

Department of Electrical and Computer Engineering, Democritus University of Thrace, Xanthi 67100, Greece

Email: tsalides@ee.duth.gr

Received 30 December 2003; Revised 18 August 2004

\begin{abstract}
A path generation method for robot-based welding systems is proposed. The method that is a modification of the method "teaching by showing" is supported by the recently developed pseudo stereovision system (PSVS). A path is generated by means of the target-object (TOB), PSVS, and the pseudo stereo visual servo control scheme proposed. A part of the new software application, called humanPT, permits the communication of a user with the robotic system. Here, PSVS, the robotic system, the TOB, the estimation of robot poses by means of the TOB, and the control and recording algorithm are described. Some new concepts concerning segmentation and point correspondence are applied as a complex image is processed. A method for calibrating the endpoint of TOB is also explained. Experimental results demonstrate the effectiveness of the proposed system.
\end{abstract}

Keywords and phrases: path generation, pseudo stereovision system, robotic application HumanPT, target-object, calibration, complex image.

\section{INTRODUCTION}

A robotic manipulator could be used in an arc-welding system. In a system like this many problems may be encountered. Some of them are (1) the movement accuracy of a torch mounted on the end effector of the robotic manipulator, (2) the generation of a proper path the torch must follow, (3) the flexibility of the system to adapt the specified path to any changes in the shape of the metal pieces to be welded, (4) the possibility of the system to change the movement rate, or the pattern of movement relatively to metal types or welding surfaces shape. The method "teaching by showing," as a method for path or trajectory generation by

This is an open access article distributed under the Creative Commons Attribution License, which permits unrestricted use, distribution, and reproduction in any medium, provided the original work is properly cited. means of a vision system, mainly assumes that a user moves the under manipulation object with his/her hand. The vision system captures images of successive scenes of the object as it is moved and then, using these images, calculates the desired path or trajectory. The different versions of this method are not easily applicable in cases where a new trajectory might be generated for a robot-based arc-welding system.

Many researchers in relevant papers propose methods for path generation by means of a vision system. In [1], Feddema and Mitchell proposed a feature-based trajectory generator. The vision module uses the location of binary image features from a camera on the robot's end effector to control the position and one degree of orientation of a manipulator. Kuniyoshi and Inaba in [2] proposed a three-camera system in which a robot learns reusable task plans by watching a human performing assembly tasks. In $[3,4,5]$, Ude and Dillmann employed the "teaching by showing" programming 
paradigm. The user specifies the desired trajectory by moving the object to be manipulated with his/her hand. The performance was measured with a stereovision system. In [6] Kang presented a system that observes a human performing the task recognizes the human grasp, and maps it onto the manipulator. In [7], Brunner et al. proposed the so-called telesensor programming concept as an easy way to program a robot off-line via learning by showing in a virtual environment. Voyles and Khosla in [8] proposed a gesturebased programming method of a robot. In [9], Johnson and Marsh presented the basic mathematical and computational framework for a new method of modeling robot manipulator workspaces. In his Ph.D. thesis [10], Sabes studied the planning of visually guided arm movements in two cases: feedback perturbation and obstacle avoidance. In [11], Gu studied the visual guidance of a robot by means of a stereovision system and using as features corner points. Päschke and Pauli in [12] implemented the programming-by-demonstration method to reconstruct a smooth 3D trajectory of a gripper, using a stereovision system. Sharma and Sutanto [13] presented a motion-planning framework by using motionplanning techniques that take into account properties of sensed data (visual feedback). Berry et al. [14] described an approach to the problem of trajectory generation by visual servoing. In [15], Ruf and Horaud proposed a methodological framework for trajectory generation in projective space. Kim et al. [16] presented a method for automatic teaching of a welding robot for free-formed seam by means of CAD data and data acquired by a laser vision sensor. In their paper [17], Zha and Du presented a new approach to the generation and optimization of the position and orientation trajectories in Cartesian task space. Simulation was made in a virtual CADbased off-line programming environment. In their recent paper Mezouar and Chaumette [18] considered a single-camera vision system mounted on a robotic manipulator. The model of the target-object is known. The camera keeps the object in the field of view and a trajectory is generated as this vision system is moving from an initial location to a final location. They examine also the case in which the object model is unknown. Finally, in their paper [19], Pachidis et al. proposed a system for path generation by means of a novel stereovision system (called pseudo stereovision system, PSVS) and complex images, which are captured from static scenes of the robot environment.

Many researchers presented also papers concerning visual servo control. Most of the visual servo control techniques are based on monocular vision systems $[20,21,22$, $23,24,25,26,27,28,29]$ and some of them are based on stereovision systems or multicamera vision systems [30, 31, $32,33,34,35]$.

The proposed system is a pose-based, endpoint closed loop (ECL), stereo visual servo control system (SVSCS) [36], in which the different locations of a moving special constructed object are recorded by means of PSVS. This different control system is called pseudo SVSCS because for first time PSVS is included in the control scheme. It uses as a target-object (TOB) a set of three planar light sources on the vertices of a triangle attached to an aluminum bar.
These three sources belong to a $5 \times 7$ dot matrix display. Their planarity has as a result the homogeneous projections to the complex image plane. Thus, as it normally happens with simple LEDs $[14,28]$, problems of diffusion or other phenomena of the projected areas do not exist. A pseudo stereovision system (PSVS) [37] is mounted on the end effector of the manipulator. An ordinary stereo system could be also used. PSVS keeps always the motion by an operator TOB in the field of view. At the same time, a predefined virtual point, with respect to the camera frame, tracks a point on the TOB (center of gravity) as it moves. In other papers, that is, [38], the end effector follows a path to track a stationary desired point or tracks it. Moreover, the current state of the TOB is calculated and recorded by the system as a state vector with respect to the world coordinate system, established on the base of the robotic manipulator Puma 761 . The operator drives the TOB along a virtual or real desired path. This is possible by means of the special constructed metal drivers; the operator can drive the TOB along a desired path, that is, a straight line, with prespecified angles that depend on the type of the metal driver. The generated path can be the path of a simple task, a part of a procedure, or the path the torch of a robot-based welding system follows. While PSVS keeps the TOB in the field of vision, in system loop cycle, state vectors can be calculated and recorded with sampling rates equal to or lower than the system loop cycle. The sampling rate is determined by means of the graphical user software interface (GUI), part of the recently developed robotic application called HumanPT. It is a complete robotic application developed in visual $\mathrm{C}++$. Its architecture is presented for the first time and can be implemented in personal computers (without the need of special hardware), permitting its use in more friendly environments. The operating system used is Microsoft Windows. Some other advantages of the proposed system are (1) easy segmentation of the three light sources image, (2) subpixel measurement accuracy, (3) robust stereo matching, (4) path generation in the working space of a robotic manipulator, and (5) model-free method.

In the sections below, PSVS is described in brief. The visual servo control scheme as well as the TOB and the calculation of state vectors are presented and analyzed. Experimental results indicate the effectiveness of the system to generate robot paths by means of the proposed pose-based pseudo stereo visual servo control method. The paper is organized as follows. In Section 2, PSVS and the related equations are briefly described. In Section 3 the robot system structure and HumanPT architecture are provided. In Section 4, details for the TOB are given and the theory related with the calculation of state vectors is provided. In Section 5, the visual servo control method is presented. Experimental results are depicted in Section 6. Finally, conclusions of this work are given in Section 7.

\section{PSVS DESCRIPTION AND CALIBRATION}

In the method proposed, the developed pseudo stereovision system (PSVS) is used as a capturing system. An ordinary 


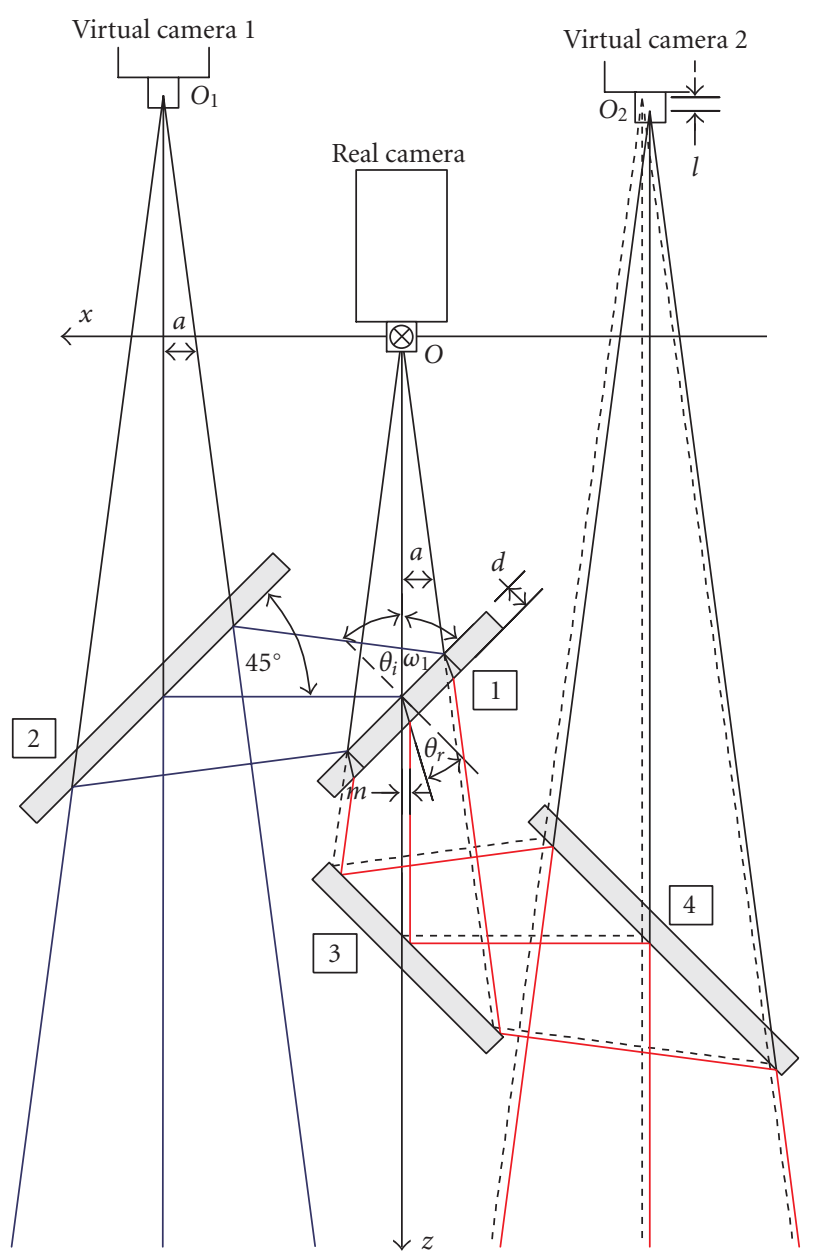

FIgURE 1: A pseudo stereovision system (PSVS) is presented. Real and virtual cameras as well as the camera coordinate system are illustrated.

stereovision system with two parallel cameras could also replace PSVS.

Details for PSVS are presented in [39]. It is a monocular stereovision system composed of a camera and four mirrors (Figure 1). The first mirror is a thin 50\% beam splitter. The other mirrors are first surface mirrors. Using PSVS a cheaper system is created combining many good features. These are (1) creation of two virtual cameras with the same geometric properties, field of view (FOV), and active focal length; (2) the angular FOV of the apparatus is the same with the FOV of the real camera; (3) it has the accuracy of an ordinary stereovision system with parallel cameras; and (4) it captures a complex image in a single slot. This complex image is the result of the superposition of the left and right images of an ordinary vision system and consequently this complex image can be processed faster than two stereo images.

The camera coordinate system (Figure 1) has as an origin the optical center $O$ of the real camera and its $z$-axis coincides with the optical axis from $O$. To calculate coordinates of path points to camera coordinate system, the intrinsic parameters as well as the distortion coefficients of the camera must be found. Here, the calibration method of Zhang [40] has been used while for corner detection the algorithm proposed in [41] has been preferred (some details are provided in the appendix).

We define the following symbols.

(i) $u_{0}, v_{0}, u_{0_{R}}, v_{0_{R}}, u_{0_{L}}, v_{0_{L}}$ are estimated coordinates of the image center in pixels of the real, the right (virtual camera 1 ), and the left cameras (virtual camera 2), respectively.

(ii) $a_{u}, a_{v}, a_{u_{R}}, a_{v_{R}}, a_{u_{L}}, a_{v_{L}}$ are estimated scale factors in image $u$ and $v$ axes, in pixels again of the real, the right (virtual camera 1), and the left cameras (virtual camera 2), respectively. axes.

(iii) $c$ is parameter describing the skew of the two image

(iv) $u_{R}, v_{R}, u_{L}, v_{L}$ are estimated coordinates of a $3 \mathrm{D}$ point in the image plane of the right (virtual camera 1) and the left cameras (virtual camera 2), respectively.

(v) $x_{0 R}, y_{0 R}, z_{0 R}$ are coordinates of the optical center $O_{1}$ with respect to the camera frame.

(vi) $x_{0 L}, y_{0 L}, z_{0 L}$ are coordinates of the optical center $\mathrm{O}_{2}$ with respect to the camera frame.

(vii) $b$ is the baseline length.

(viii) $m$ is parameter describing the parallel displacement of the virtual optical axis from $\mathrm{O}_{2}$ along $x$-axis due to refraction phenomena in mirror (1).

(ix) $l$ is parameter describing the shifting of the optical center $\mathrm{O}_{2}$ along the optical axis (Figure 1) due to refraction phenomena in mirror (1).

(x) $\omega_{1}=90^{\circ}-\theta_{i}$ is angle forming the optical axis with a mirror plane $\left(\theta_{i}\right.$ is the incidence angle).

(xi) $\theta_{r}$ is refraction angle (Figure 1 ).

(xii) $d$ is the thickness of mirror (1).

The transformation matrix with the intrinsic camera parameters is

$$
A=\left[\begin{array}{ccc}
a_{u} & c & u_{0} \\
0 & a_{v} & v_{0} \\
0 & 0 & 0
\end{array}\right] .
$$

The equations giving the coordinates of a point $P\left(x_{P}, y_{P}, z_{P}\right)$ in 3D space, in a general form, for parallel cameras located in place of virtual cameras, are

$$
\begin{aligned}
x_{P}= & \frac{\left(u_{R}-u_{0 R}\right)}{a_{u R}} \cdot\left(z_{P}-z_{0 R}\right)+x_{0 R}, \\
x_{P}= & \frac{\left(u_{L}-u_{0 L}\right)}{a_{u L}} \cdot\left(z_{P}-z_{0 L}\right)+x_{0 L}, \\
y_{P}= & \frac{\left(v_{R}-v_{0 R}\right)}{a_{v R}} \cdot\left(z_{P}-z_{0 R}\right)+y_{0 R}, \\
y_{P}= & \frac{\left(v_{L}-v_{0 L}\right)}{a_{v L}} \cdot\left(z_{P}-z_{0 L}\right)+y_{0 L}, \\
z_{P}= & \frac{a_{u L} \cdot\left(u_{R}-u_{0 R}\right) \cdot z_{0 R}-a_{u R} \cdot\left(u_{L}-u_{0 L}\right) \cdot z_{0 L}}{a_{u L} \cdot\left(u_{R}-u_{0 R}\right)-a_{u R} \cdot\left(u_{L}-u_{0 L}\right)} \\
& +\frac{a_{u L} \cdot a_{u R} \cdot\left(x_{0 L}-x_{0 R}\right)}{a_{u L} \cdot\left(u_{R}-u_{0 R}\right)-a_{u R} \cdot\left(u_{L}-u_{0 L}\right)} .
\end{aligned}
$$


The above equations are applied to PSVS and, using the simplifications

$$
\begin{gathered}
a_{u L}=a_{u R}=a_{u}, \quad a_{v L}=a_{v R}=a_{v}, \\
x_{0 L}=-\frac{b}{2}-m, \quad y_{0 L}=0, \quad z_{0 L}=-\frac{b}{2}+l, \\
x_{0 R}=\frac{b}{2}, \quad y_{0 R}=0, \quad z_{0 R}=-\frac{b}{2},
\end{gathered}
$$

are modified to the following equations:

$$
\begin{aligned}
& x_{P}=\frac{1}{a_{u}} \cdot\left(z_{P}+\frac{b}{2}\right) \cdot\left(u_{R}-u_{0 R}\right)+\frac{b}{2}, \\
& x_{P}=\frac{1}{a_{u}} \cdot\left(z_{P}+\frac{b}{2}-l\right) \cdot\left(u_{L}-u_{0 L}\right)-\frac{b}{2}-m, \\
& y_{P}=\frac{1}{a_{v}} \cdot\left(z_{P}+\frac{b}{2}\right) \cdot\left(v_{R}-v_{0 R}\right), \\
& y_{P}=\frac{1}{a_{v}} \cdot\left(z_{P}+\frac{b}{2}-l\right) \cdot\left(v_{L}-v_{0 L}\right), \\
& z_{P}=\frac{a_{u} \cdot(b+m)+l \cdot\left(u_{L}-u_{0 L}\right)}{\left(u_{L}-u_{0 L}\right)-\left(u_{R}-u_{0 R}\right)}-\frac{b}{2} .
\end{aligned}
$$

In (4), parameter $m$ is derived as

$$
m=\frac{d \cdot \cos \left(\omega_{1}+\theta_{r}\right)}{\cos \theta_{r}}
$$

while the parameter $l$ is derived as

$$
l=\frac{d}{\cos \theta_{r}}\left(1-\sin \left(\omega_{1}+\theta_{r}\right)+\cos \left(\omega_{1}+\theta_{r}\right) \cdot \tan \omega_{1}\right)
$$

For mirror (1) thickness $d$ equals $1 \mathrm{~mm}$ (as in PSVS), $m=$ $0.329 \mathrm{~mm}$, and $l=0.378 \mathrm{~mm}$ (refraction indices $n_{\text {glass }}=1,5$ and $\left.n_{\text {air }}=1\right)$.

\section{SYSTEM STRUCTURE AND HUMANPT ARCHITECTURE}

The experimental system used consists of a Puma 761 robotic manipulator and two personal computers. PSVS is mounted on the end effector of the robotic manipulator. The first computer communicates with the Puma robot controller through the available ALTER port. This is a special serial port for real-time communication $(28 \mathrm{~ms} /$ cycle). The baud rate has been determined at 38400 bps making some changes in the serial board of the Puma controller (the standard and the unique baud rate, as it has been determined by Unimation, was $19200 \mathrm{bps}$ ). Even though Windows operating systems are used (it is not a real-time operating system; there are problems with latencies), a stable communication, in realtime, between the controller and the server was achieved. The second computer is used as a client computer and communicates with the server through Ethernet. These devices (PCs and the robot controller) cooperate robustly and reliably by means of the robotic application HumanPT (Figure 2). It has been developed in visual $\mathrm{C}++$ (more than 70000 lines of code).

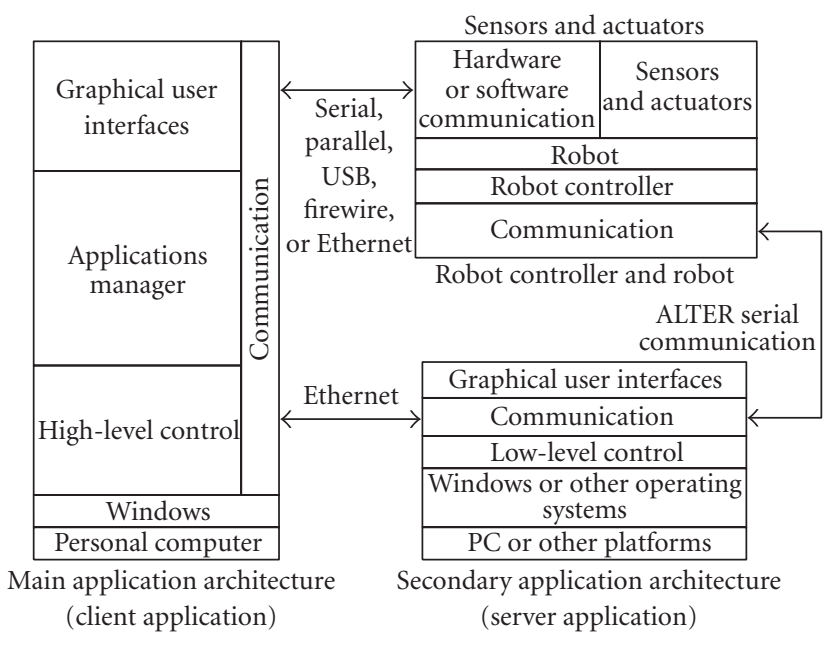

Figure 2: HumanPT architecture.

The application is executed in both computers. In the first one, a light version of it permits the reliable and robust communication of the PC with the robot controller. At the same time, it permits the low-level control of the Puma robot through ALTER port and the communication through Ethernet with the client computer. In the client computer, the full version is executed. Some features of the full version are (a) communication with the server computer through Ethernet, (b) calibration of cameras, (c) hand-eye calibration, (d) mirrors alignment check, (e) methods for path generation by means of a vision system, (f) control of the manipulator by means of several sources of data, and ( $\mathrm{g}$ ) real-time control by using visual servoing.

\section{TARGET-OBJECT DESCRIPTION AND POSE ESTIMATION}

The objective of this section is to calculate initially the state vector of a point $G$ (and of the frame attached to it) that belongs to TOB.

The state vector of it must be calculated robustly and easily by means of PSVS or a stereovision system. Light conditions (or luminosity changes) must not affect the correct extraction of features from images captured. A right-hand rectangular coordinate system is established with origin the point $G$ (TOB frame). To determine the pose of this frame with respect to the camera frame, it is necessary that at least three points be found on the TOB (Figure 3c). These points are selected to be the geometric centers of the circular areas of the three light sources. Each source of the targetobject is located on a vertex of the triangle. The origin $G$ of TOB frame is located on the center of gravity of the centers of the three light sources. While the above light sources can be randomly located on a planar surface, constructing the TOB, these sources are selected to be on vertices of an isosceles or equilateral triangle. Thus, it is easier to geometrically determine the location of the frame attached to the TOB. 


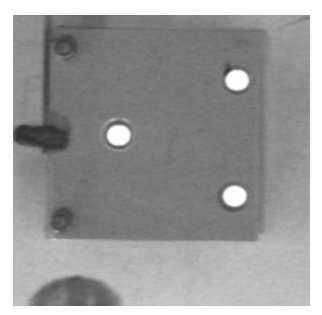

(a)

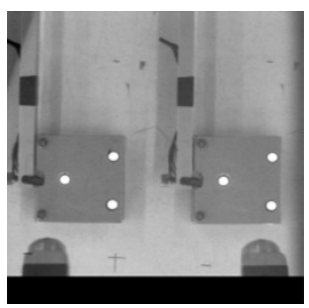

(b)

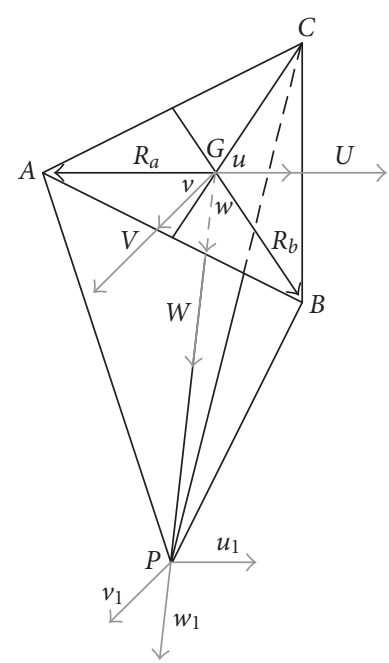

(c)

Figure 3: Pictures and the schematic diagram of the target-object. (a) Top view of the TOB in which three dots of the dot display are depicted. (b) A complex image that has been captured by means of PSVS. (c) Schematic diagram of the TOB with the coordinate systems. $A, B$, and $C$ are the centers of the three light sources while $P$ (endpoint of a metal driver) is found at the invisible side of the TOB.

Moreover, to increase measurements accuracy, these sources are selected to be planar. Instead of using three simple LEDs, a $5 \times 7$ dot matrix display has been used. The selection of the sources location (dots) is made by means of an electronic circuit or an aluminum mask adapted on the display. The TOB, as it is already presented, is mounted on an aluminum bar (Figure $4 \mathrm{a}$ ). The coordinates of the origin $G\left(g_{x}, g_{y}, g_{z}\right)$ with respect to the camera frame are the mean values of the coordinates of the three points $A\left(a_{x}, a_{y}, a_{z}\right)$, $B\left(b_{x}, b_{y}, b_{z}\right), C\left(c_{x}, c_{y}, c_{z}\right)$. Then the vectors $\mathbf{R}_{a}\left(r_{a x}, r_{a y}, r_{a z}\right)$ and $\mathbf{R}_{b}\left(r_{b x}, r_{b y}, r_{b z}\right)$ are defined as $\mathbf{R}_{a}=\mathbf{G A}$ and $\mathbf{R}_{b}=\mathbf{G B}$ (Figure 3c). The vertical vector $\mathbf{W}\left(W_{x}, W_{y}, W_{z}\right)$ to the plane with origin $G$ is given as $\mathbf{W}=\mathbf{R}_{b} \times \mathbf{R}_{a}$, while the vector $\mathbf{U}\left(U_{x}, U_{y}, U_{z}\right)$ is defined to be equal to $-\mathbf{R}_{a}$, that is, $\mathbf{U}=-\mathbf{R}_{a}$. Then, the third vector $\mathbf{V}\left(V_{x}, V_{y}, V_{z}\right)$ is computed as the cross product of the two previous vectors, $\mathbf{V}=\mathbf{W} \times \mathbf{U}$. The three vectors created in this way are vertical to each other and have the same origin $G\left(g_{x}, g_{y}, g_{z}\right)$. The unit vectors derived from them are

$$
\mathbf{u}=\frac{\mathbf{U}}{|\mathbf{U}|}, \quad \mathbf{v}=\frac{\mathbf{V}}{|\mathbf{V}|}, \quad \mathbf{w}=\frac{\mathbf{W}}{|\mathbf{W}|},
$$

and compose a rectangular coordinate system attached to the point $G$ (TOB frame). If the rotation matrices, derived by the above three unit vectors and the coordinates of the origin with respect to the camera frame are of the form ${ }^{C} \mathbf{R}_{G}=[\mathbf{u v w}]$ and ${ }^{C} \mathbf{t}_{G}=\left[g_{x}, g_{y}, g_{z}\right]^{T}$, respectively, then the homogeneous matrix with respect to the camera frame is of

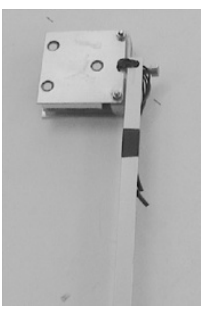

(a)

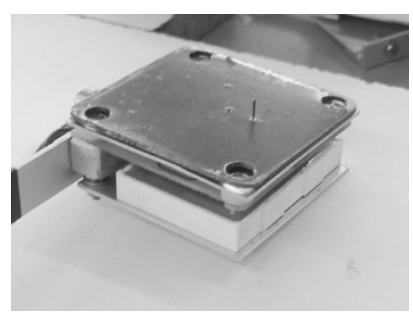

(b)

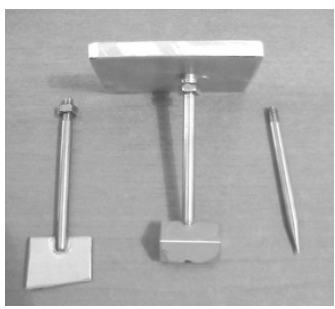

(c)

FIgure 4: (a) The TOB is attached to an aluminum bar. (b) Bottom side; the dot matrix display and the needle attached to the bottom side of the TOB stainless needle are distinguished. (c) Different types of TOB drivers that can be attached to the bottom side of the TOB. Thus, for instance, the operator can drive the TOB along an edge (a line) with a constant angle.

the form

$$
{ }^{C} \mathbf{T}_{G}=\left[\begin{array}{cc}
{ }^{C} \mathbf{R}_{G} & { }^{C} \mathbf{t}_{G} \\
\mathbf{0}^{T} & 1
\end{array}\right] .
$$

In each cycle, a gray scale image $\mathbf{I}(i, j)$ is captured by using PSVS and is converted to a binary image with a prespecified threshold ( $\mathrm{T}$ ) by means of the software and the graphical user interface, which are parts of HumanPT (Figure 5).

The binary image $\mathbf{I}_{b}(i, j)$ is inverted to the final image $\mathbf{I}_{\text {in }}(i, j)$, which contains only six black circular areas. Segmentation of each circular area is integrated in two steps. First this image is convoluted with the cross-type $9 \times 9$ template $\mathbf{M}(k, l)$ to find a seed for a circular area. A seed is defined as a gray or color pixel (different for each shape) with the property to be the center of a cross-shaped template (Figure 6), formed with black pixels (" $C$ "). In this way, noise pixels are rejected and the convolution procedure is faster because of the reduced number of pixels used in the template [41] (see the appendix for some details). Seventeen pixels of the $9 \times 9$ template are convoluted instead of eighty-one. In the second step the seed 


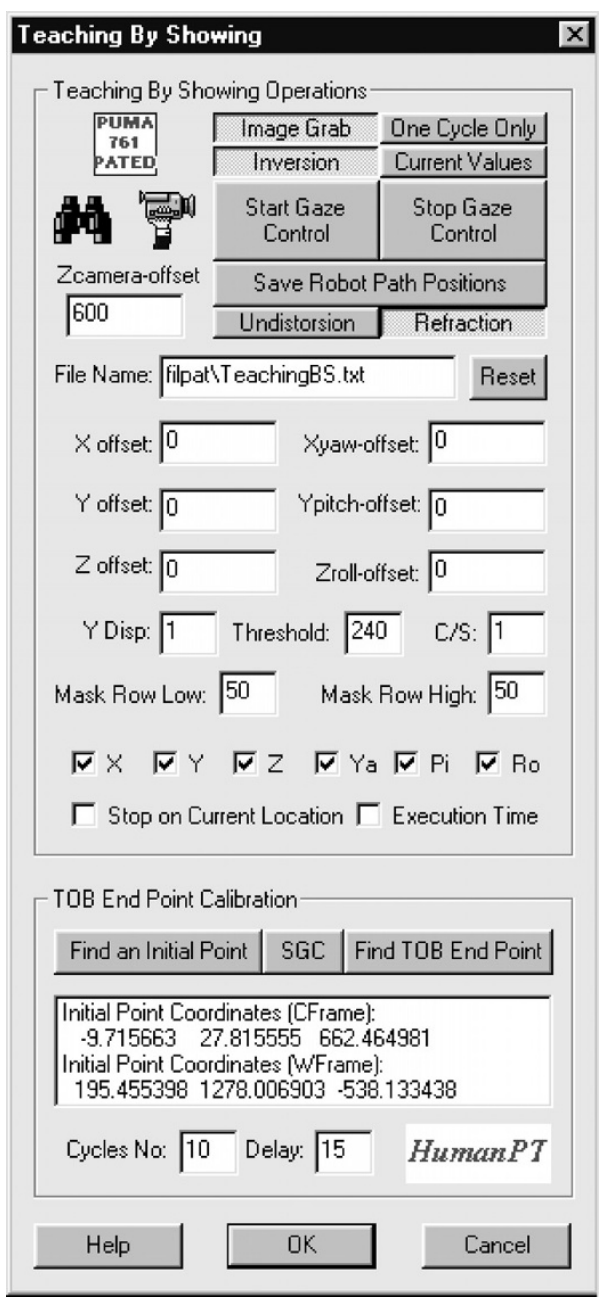

FIgURE 5: The graphical user interface (GUI) used in the proposed method.

is propagated to the whole shape (the black circular area), scanning in a small area with a center this seed that includes the circular area. The procedure is repeated until all the circular shapes are segmented. For three light sources, six circular shapes are appearing in the complex image captured by means of PSVS (Figure 3b). The algorithm finds the centers of these areas with subpixel accuracy. The correspondence problem is limited to three pairs of points (the centers of the circular areas). During system operation, only two points are found in the same scan line (epipolar line), which are the two different views of the centers of a circular area in the complex image. Thus, each pair is detected, its points are examined, and, if necessary, are set in correct order. From these three pairs, by means of (4), coordinates of each 3D point with respect to the camera frame are calculated.

We define matrix ${ }^{F} \mathbf{T}_{C}$ as the homogeneous matrix arising from the solution of hand-eye calibration problem [42], found with a previous procedure and matrix ${ }^{W} \mathbf{T}_{F}$ as the homogeneous matrix giving the location of the flange of the manipulator with respect to the world frame. The last ma-

\begin{tabular}{|c|c|c|c|c|c|c|c|c|}
\hline$X$ & $X$ & $X$ & $X$ & $C$ & $X$ & $X$ & $X$ & $X$ \\
\hline$X$ & $X$ & $X$ & $X$ & $C$ & $X$ & $X$ & $X$ & $X$ \\
\hline$X$ & $X$ & $X$ & $X$ & $C$ & $X$ & $X$ & $X$ & $X$ \\
\hline$X$ & $X$ & $X$ & $X$ & $C$ & $X$ & $X$ & $X$ & $X$ \\
\hline$C$ & $C$ & $C$ & $C$ & $C$ & $C$ & $C$ & $C$ & $C$ \\
\hline$X$ & $X$ & $X$ & $X$ & $C$ & $X$ & $X$ & $X$ & $X$ \\
\hline$X$ & $X$ & $X$ & $X$ & $C$ & $X$ & $X$ & $X$ & $X$ \\
\hline$X$ & $X$ & $X$ & $X$ & $C$ & $X$ & $X$ & $X$ & $X$ \\
\hline$X$ & $X$ & $X$ & $X$ & $C$ & $X$ & $X$ & $X$ & $X$ \\
\hline
\end{tabular}

FIGURE 6: Seeds can be found by means of the illustrated cross-type $9 \times 9$ template. " $C$ ": black pixels. " $X$ ": don't care pixels.

trix is provided in each cycle ( 28 milliseconds) by the system using the ALTER communication port at 38400 bps. Then the coordinates of a point with respect to the world frame, established on the base of the Puma 761 robotic manipulator, are derived by means of the transformation matrix ${ }^{W} \mathbf{T}_{G}$ which equals ${ }^{W} \mathbf{T}_{G}={ }^{W} \mathbf{T}_{F} \cdot{ }^{F} \mathbf{T}_{C} \cdot{ }^{C} \mathbf{T}_{G}$. Euler angles yaw, pitch, and roll are computed from the final rotation matrix ${ }^{W} \mathbf{R}_{G}$. Thus a six-component vector expressing the state of the TOB frame is created. If $\left(x_{W G}, y_{W G}, z_{W G}\right)$ are the coordinates of point $G$ with respect to the world frame and $\left(\psi_{W G}, \theta_{W G}, \varphi_{W G}\right)$ are Euler angles yaw, pitch, and roll, then this vector is $\mathbf{X}_{W G}=\left(x_{W G}, y_{W G}, z_{W G}, \psi_{W G}, \theta_{W G}, \varphi_{W G}\right)^{T}$ and it is stored in a file.

The basic steps of the algorithm used are the following.

(1) Capture a complex image.

(2) Convert to a binary image and then mask the binary image (if necessary).

(3) Invert this image (if this property has been selected).

(4) Automatically put a seed to each shape, propagate it, and find the mean value of shape pixels location (subpixel accuracy).

(5) Calculate the coordinates of points in 3D space.

(6) Calculate the three vectors of the TOB frame.

(7) Calculate the homogeneous matrix ${ }^{C} \mathbf{T}_{G}$.

(8) Find the transformation matrix of the TOB frame with respect to the world frame by means of the product ${ }^{W} \mathbf{T}_{G}={ }^{W} \mathbf{T}_{F} \cdot{ }^{F} \mathbf{T}_{C} \cdot{ }^{C} \mathbf{T}_{G}$.

(9) Find from the final rotation matrix, ${ }^{W} \mathbf{R}_{G}$, Euler angles yaw, pitch, and roll $\left(\psi_{W G}, \theta_{W G}, \varphi_{W G}\right)$.

(10) Create the six-component vector with the position and orientation of the TOB frame that is equal to $\mathbf{X}_{W G}=$ $\left(x_{W G}, y_{W G}, z_{W G}, \psi_{W G}, \theta_{W G}, \varphi_{W G},\right)^{T}$ and store the vector.

(11) Calculate the new desired location.

(12) Repeat steps 1-11.

By means of the above procedure, the desired robot path is generated in cases where the TOB does not follow a given edge and then the previous calculations and the related algorithm provide successive path points. Each estimated vector represents the current state of the TOB frame. But point $G$ cannot be always a desired path point. In cases where the 
TOB follows a given edge, we assume that the point of interest (point of the desired path) is found initially along with the straight line passing from point $G$, vertically to the surface of the light sources at the invisible side (bottom side) of the TOB (Figures 3c, $4 \mathrm{~b}$ ). By construction, this point is the endpoint of a metal driver (i.e., a needle) attached to the TOB. In Figure 4c some types of metal drivers are illustrated. By means of the proper metal driver, the operator can drive the TOB along a line with a prespecified angle or angles (i.e., $15^{\circ}$ ). This angle could be, for instance, the angle the torch forms with a welding line. Thus, an operator can drive the endpoint of the driver attached to the TOB along the edges of an object and then the recorded state vectors of that point correspond to points of a desired path. Let this point be the point $P\left(p_{x}, p_{y}, p_{z}\right)$ (Figure $\left.3 \mathrm{c}\right)$. It will be the forth vertex of a pyramid. The other three vertices are the geometric centers of the light sources. To calculate the state vector of point $P$, we define first a frame with that point an origin. Let ${ }^{G} \mathbf{R}_{P}$ represent the rotation matrix that rotates the $u_{1} v_{1} w_{1}$ frame (origin $P$ ) with respect to the $u v w$ frame (origin $G$ ) and let vector ${ }^{G} \mathbf{t}_{P}$ represent the translation of point $P$ with respect to point $G$. The homogeneous matrix transforming the point $P$ frame to the TOB frame is of the form

$$
{ }^{G} \mathbf{T}_{P}=\left[\begin{array}{cc}
{ }^{G} \mathbf{R}_{P} & { }^{G} \mathbf{t}_{P} \\
0 & 1
\end{array}\right] .
$$

By definition the frame attached to point $P$ is parallel to the TOB frame. Then the matrix ${ }^{G} \mathbf{R}_{P}$ equals the unit matrix I, namely, ${ }^{G} \mathbf{R}_{P}=$ I. If point $P$ is found along $w$-axis, then ${ }^{G} \mathbf{t}_{P}=$ $(0,0, d)^{T}$, where $d$ is the Euclidean distance between points $P$ and $G$. In this case the location of the endpoint $P$ can be estimated without any calibration of the TOB and the metal driver adapted on it, by means of the existing geometry.

In the general case, the relative location of the frame attached to $P$ with respect to the TOB frame is estimated by means of the calibration procedure proposed below.

\subsection{Calibration of the driver attached to the TOB}

To estimate the location of the point $P$ with respect to the geometric center $G$ of the TOB, first, the coordinates of a 3D point $P^{\prime}\left(p_{x}^{\prime}, p_{y}^{\prime}, p_{z}^{\prime}\right)$ are estimated with respect to the world frame by means of PSVS. Let the vector ${ }^{C} \mathbf{t}_{p^{\prime}}$ be estimated by means of PSVS and (4). Then, the homogeneous matrix ${ }^{W} \mathbf{t}_{P^{\prime}}$ is calculated as

$$
{ }^{W} \mathbf{t}_{p^{\prime}}={ }^{W} \mathbf{T}_{F} \cdot{ }^{F} \mathbf{T}_{C} \cdot{ }^{C} \mathbf{t}_{p^{\prime}} \cdot
$$

Point $P^{\prime}$ coordinates are accurately estimated as the center of the pattern of a 2D object (i.e., a circle, a rectangle, or a square area in which the location of the geometric center (GC) is found easily). This point is computed with subpixel accuracy for each view in image coordinates and then, by implementing PSVS equations, point coordinates with respect to the world frame are calculated. This procedure is repeated for a number $N$ of different poses of the robotic manipulator and PSVS mounted on it. The mean values of these coordinates provide the coordinates of the point $P^{\prime}$ with respect to the world frame

$$
{ }^{W} \mathbf{t}_{P^{\prime}}=\frac{1}{N} \sum{ }^{W} \mathbf{t}_{P^{\prime} i}
$$

Then, coordinates of point $P$ with respect to TOB frame are calculated by locating the endpoint of the TOB driver on the GC of the pattern (point $P^{\prime}$ ). The proposed visual servo control algorithm is set in operation and the operator, retaining this contact of the endpoint of the TOB driver on the GC of the pattern, rotates the TOB. After an initial translation and rotation of PSVS to approach and keep the TOB in the proper view, PSVS tracks the TOB while at the same time the different states of the point $G$ and the frame attached to it are recorded. For each state, the vector providing the coordinates of point $P$ with respect to point $G,{ }^{G} \mathbf{t}_{P}$, is estimated by the following multiplications and matrices inversions:

$$
\begin{aligned}
{ }^{W} \mathbf{t}_{P} & ={ }^{W} \mathbf{T}_{F} \cdot{ }^{F} \mathbf{T}_{C} \cdot{ }^{C} \mathbf{T}_{G} \cdot{ }^{G} \mathbf{t}_{P} \Longrightarrow \\
{ }^{G} \mathbf{t}_{P} & ={ }^{C} \mathbf{T}_{G}^{-1} \cdot{ }^{F} \mathbf{T}_{C}^{-1} \cdot{ }^{W} \mathbf{T}_{F}^{-1} \cdot{ }^{W} \mathbf{t}_{P} .
\end{aligned}
$$

The vector ${ }^{W} \mathbf{t}_{P} \equiv{ }^{W} \mathbf{t}_{p^{\prime}}$ and the matrix ${ }^{F} \mathbf{T}_{C}$ have been estimated by the already mentioned procedure while matrices ${ }^{C} \mathbf{T}_{G}$ and ${ }^{W} \mathbf{T}_{F}$ are provided for each loop cycle. To find vector ${ }^{G} \mathbf{t}_{P}$ as accurately as possible, if $N$ represents the number of the different vectors recorded by the system, it is calculated as the mean value of the estimated vectors by means of the above procedure, that is,

$$
{ }^{G} \mathbf{t}_{P}=\frac{1}{N} \sum{ }^{G} \mathbf{t}_{P i}
$$

The number $N$ of vectors is prespecified by means of the GUI (Figure 5, bottom).

\section{PSEUDO STEREO VISUAL SERVO CONTROL}

As it has already been mentioned, the control method used is a pose-based pseudo stereo visual control method. The term pseudo is used because one complex image per cycle is processed. To implement an endpoint closed-loop (ECL) visual servoing, two points (a real and a desired point) and the frames attached to them are considered. The first frame is the TOB frame. Without loss of generality, we consider as desired point a virtual point in 3D space, $D\left(d_{x}, d_{y}, d_{z}\right)$, found on the $z$-axis $\left(d_{x}=0, d_{y}=0\right)$ of the camera frame, and the frame attached to it is parallel to the camera frame. Thus, the distance between the TOB frame and the camera frame can be regulated by means of the GUI. As PSVS moves, it keeps the TOB in the field of view while the virtual point tracks TOB's frame origin. In the general case, the virtual desired point can be selected so that its double track on the image plane exists. When the tool tip (mounted on the end effector of the manipulator) tracks a Cartesian path, the desired point frame can be the tool tip frame. Poses of the above frames are estimated with respect to the robot flange frame. Let ${ }^{C} \mathbf{T}_{D}$ 
be the homogeneous matrix giving the pose of the desired point $D$ frame to the camera frame. The homogeneous matrices ${ }^{F} \mathbf{T}_{G},{ }^{F} \mathbf{T}_{D}$ giving the poses of the TOB frame and the desired point $D$ frame to the robot flange frame are derived by means of the following products: ${ }^{F} \mathbf{T}_{G}={ }^{F} \mathbf{T}_{C} \cdot{ }^{C} \mathbf{T}_{G}$ and ${ }^{F} \mathbf{T}_{D}=$ ${ }^{F} \mathbf{T}_{C} \cdot{ }^{C} \mathbf{T}_{D}$. If $\left(x_{F G}, y_{F G}, z_{F G}\right),\left(x_{F D}, y_{F D}, z_{F D}\right)$ are coordinates of points $G$ and $D$, respectively, to the flange frame and $\left(\psi_{F G}, \theta_{F G}, \varphi_{F G}\right),\left(\psi_{F D}, \theta_{F D}, \varphi_{F D}\right)$ are the related Euler angles (yaw, pitch, and roll) to the camera frame, then the current state vector $\mathbf{X}_{F G}=\left(x_{F G}, y_{F G}, z_{F G}, \psi_{F G}, \theta_{F G}, \varphi_{F G}\right)^{T}$ and the desired state vector $\mathbf{X}_{F D}=\left(x_{F D}, y_{F D}, z_{F D}, \psi_{F D}, \theta_{F D}, \varphi_{F D}\right)^{T}$ are calculated with respect to the robot flange frame by means of these matrices. Then, a kinematic error function with respect to the flange frame can be simply written as $\mathbf{E}_{F}=\mathbf{X}_{F G}-\mathbf{X}_{F D}$. The relation is valid when small changes in angles per cycle are appearing and in this case angles yaw, pitch, and roll may be used as a $3 \times 1$ vector [43]. The low-level control algorithm implemented in the robot system through ALTER port determines the maximum limits for safe and reliable operation of the robotic manipulator. Thus the above constraint for small angle changes/cycle is always valid. Let $\mathbf{K}$ be a $6 \times 6$ weight matrix. Then the $6 \times 1$ kinematic screw of the robot flange is $\mathbf{V}_{F}=-\mathbf{K} \cdot \mathbf{E}_{F}$.

\section{EXPERIMENTAL RESULTS}

The robot path generation method described above has been implemented by means of a Puma 761 robotic manipulator and two PCs (running at $350 \mathrm{MHz}$ ). The graphical user interface (GUI) permits offset regulation of the distance $d_{z}$ of PSVS (mounted on the end effector of the robotic manipulator) from the virtual point. An operator can also select some more operations like inversion of images, threshold selection, recording delay (sampling rate), and saving facilities.

The low-level step controller of the system is regulated to maximum speed values of $100 \mathrm{~mm} / \mathrm{s}$ and $60 \mathrm{deg} / \mathrm{s}$ for translation and rotation, respectively. The recording delay was defined to 10 cycles/vector. The measured cycle of the servo loop (control + recording) was 70 milliseconds. To show the accuracy and the precision of the system, the TOB remains stationary and the robot system is activated. After an initial translation and rotation of PSVS (of the virtual point) to track the TOB, it continues to track the TOB, while at the same time state vectors are recorded (106 vectors). In Figures $7 \mathrm{a}, 7 \mathrm{~b} y$ versus $x$ and $z$ versus $y$ are illustrated, respectively.

The real TOB state with respect to the world frame is the following: $x_{W G}=236.132 \mathrm{~mm}, y_{W G}=1248.772 \mathrm{~mm}$, $z_{W G}=85.284 \mathrm{~mm}, \psi_{W G}=-2.598^{\circ}, \theta_{W G}=-0.883^{\circ}, \varphi_{W G}=$ $-4.535^{\circ}$. It was accurately estimated by means of PSVS. Then by using the recorded state vectors, the mean error (ME), the mean absolute error (MAE), the maximum error (MaxE), and the standard deviation (SD) are calculated. The results are presented in Table 1 .

To show the accuracy of the system as the TOB moves, the TOB with its aluminum bar is attached to a rotated base (Figure 8a). Figure 8a shows also PSVS mounted on the end effector of the robotic manipulator. The coordinates of the center of this base (in $\mathrm{mm}$ ) with respect to the world frame

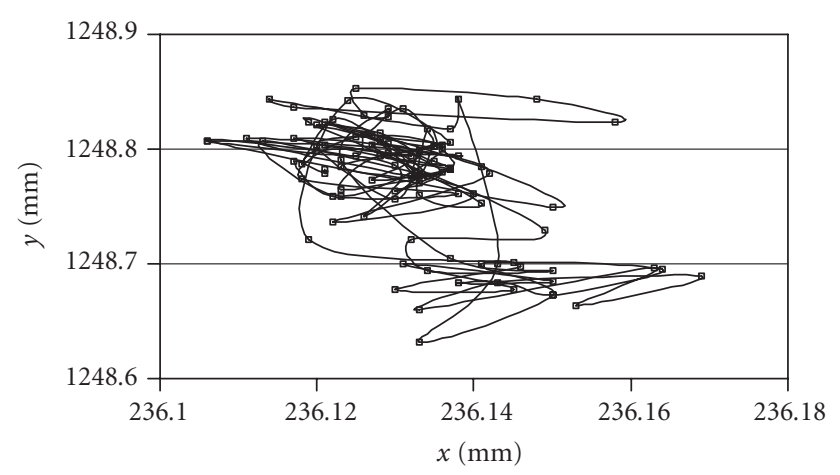

(a)

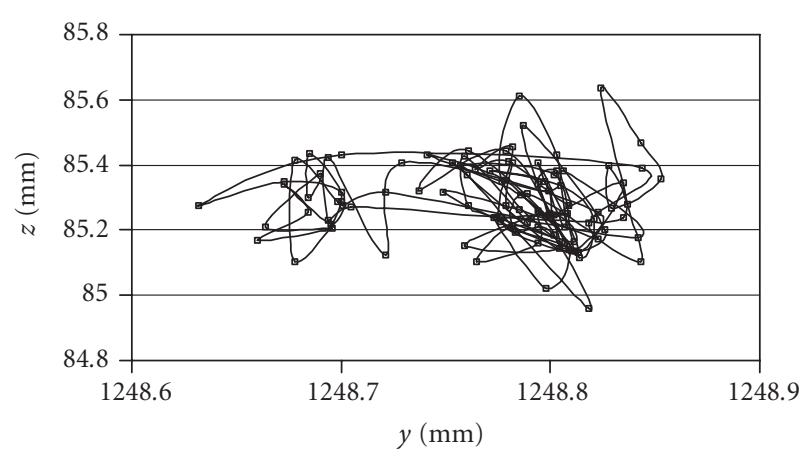

(b)

FIGURE 7: (a) Graph of $y$ versus $x$ and (b) $z$ versus $y$ as PSVS tracks the stationary TOB. Coordinates are considered with respect to the world frame.

are the following: $\left(x_{b}, y_{b}, z_{b}\right)=(147.5,1378.5,448.0)$ while the radius of the circle is $246 \mathrm{~mm}$. The distance $d_{z}$ is determined to be $d_{z}=600 \mathrm{~mm}$. The initial angle indicated by the base angle index is $50^{\circ}$. The robot system is activated and PSVS (the virtual point) tracks the TOB in its initial location. The operator rotates the TOB while the state vectors are recorded. PSVS keeps the TOB in the field of view. The system stops when the base index is about $-50^{\circ}$. In Figure $8 \mathrm{~b}$ the generated arc is depicted while in Figures $8 \mathrm{c}$ and $8 \mathrm{~d}$ the variation of the position and of the rotation angles are illustrated, respectively. From Figure $8 \mathrm{~d}$ it is concluded that angle changes are less than $\pm 1.5^{\circ}$. By means of the state vectors recorded, the mean error (ME), the mean absolute error (MAE), and the maximum error (MaxE) are calculated. The results are presented in Table 2.

As a third experiment, we drove the TOB along the edges of a trapezium-shaped aluminum piece. Its dimensions are $b_{1}=451 \mathrm{~mm}, b_{2}=361 \mathrm{~mm}$, and $h=45 \mathrm{~mm}$. First the endpoint of the needle (the driver attached to the TOB) is estimated. The path generated is illustrated in Figure 9a. In Table 3, as mentioned previously, the calculated errors are presented. These errors are increased because the operator cannot drive accurately (without using the correct metal driver) the TOB around areas where abrupt changes of edge direction are observed (i.e., vertices). 
TABLE 1: Errors and standard deviation as PSVS tracks the TOB found in a static location (mm).

\begin{tabular}{l|cccccc}
\hline Error & $x_{W G}$ & $y_{W G}$ & $z_{W G}$ & $\psi_{W G}$ & $\theta_{W G}$ & $\varphi_{W G}$ \\
\hline ME & $1.13 \mathrm{E}-13$ & $-4.3 \mathrm{E}-15$ & $2.44 \mathrm{E}-14$ & $-6.1 \mathrm{E}-16$ & $1.0 \mathrm{E}-15$ & $-8.8 \mathrm{E}-15$ \\
MAE & 0.0089 & 0.0412 & 0.0949 & 0.0056 & 0.0026 & 0.0039 \\
MaxE & 0.0369 & 0.1403 & 0.352 & 0.2972 & 0.1401 & 0.2041 \\
SD & 0.0116 & 0.0508 & 0.1170 & 0.0290 & 0.0137 & 0.0199 \\
\hline
\end{tabular}

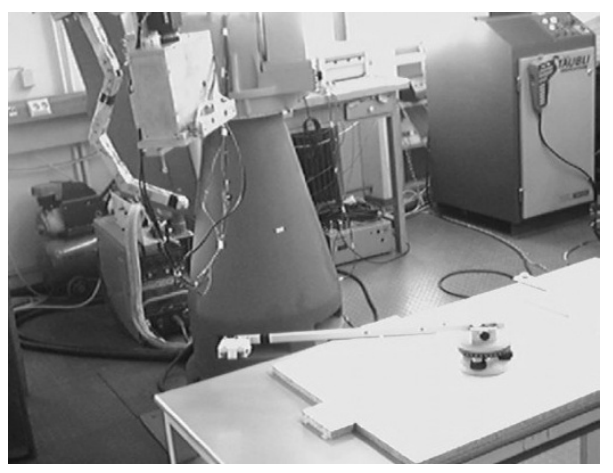

(a)

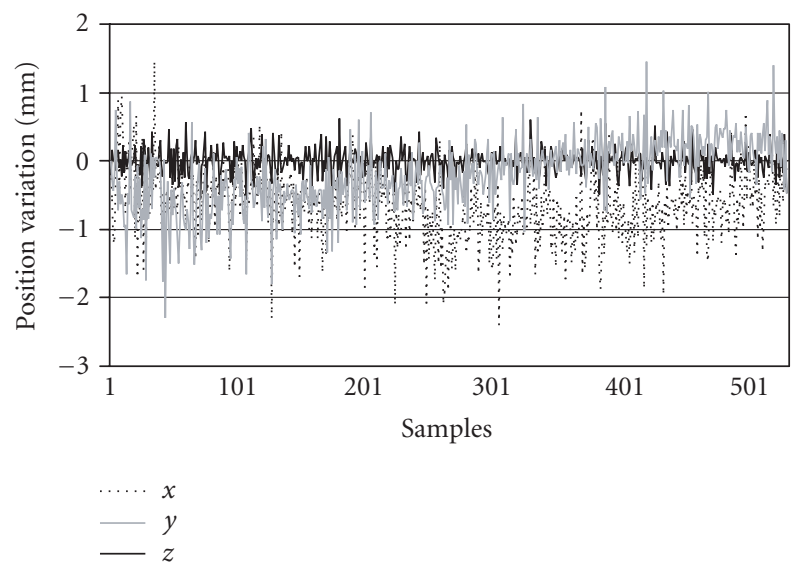

(c)

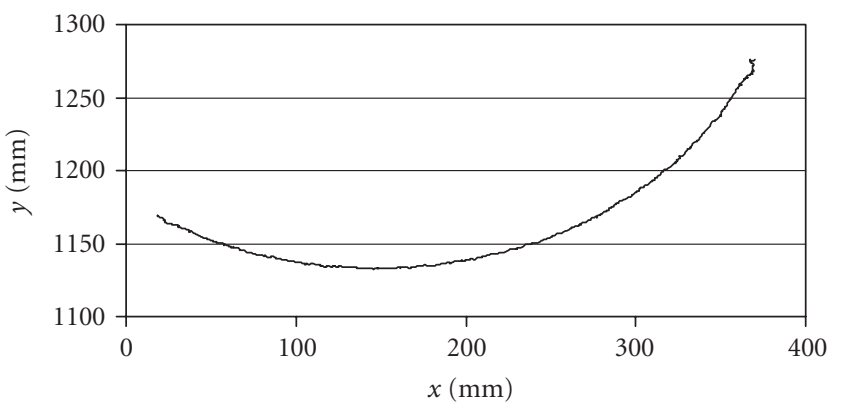

(b)

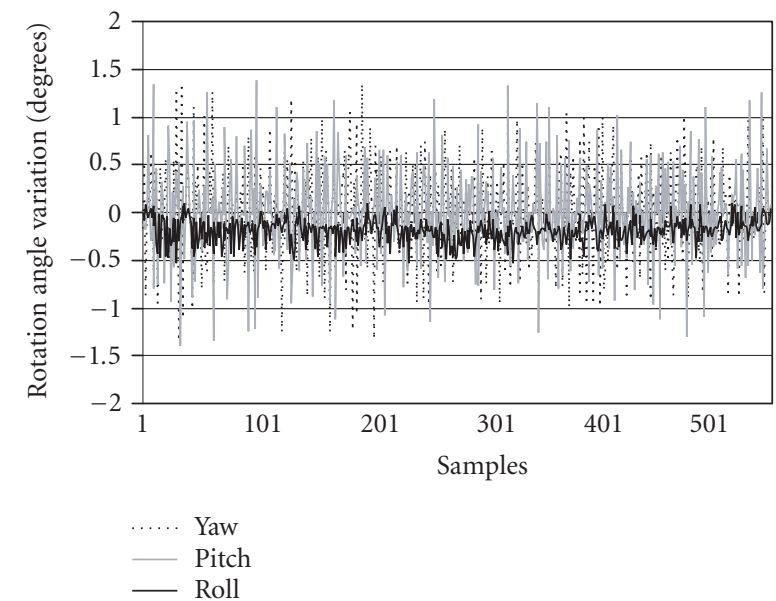

(d)

FIgURE 8: (a) A picture of the robotic system. (b) The graph of $y$ versus $x$. (c) Variation of the position of the TOB. (d) Variation of the rotation angles of the TOB. Coordinates in Figures $8 \mathrm{~b}, 8 \mathrm{c}, 8 \mathrm{~d}$ are considered with respect to the world frame.

TABLE 2: Errors as PSVS tracks the TOB following a circular path (mm).

\begin{tabular}{l|ccccrr}
\hline Error & $x_{W G}$ & $y_{W G}$ & $z_{W G}$ & $\psi_{W G}$ & $\theta_{W G}$ & $\varphi_{W G}$ \\
\hline ME & 0.0436 & 0.1093 & -0.294 & 0.1795 & -0.0472 & -0.1662 \\
MAE & 0.2466 & 0.4843 & 0.3048 & 0.6212 & 0.4581 & 0.4313 \\
MaxE & 1.1477 & 1.7052 & 1.0210 & 1.8740 & 1.7380 & 0.8604 \\
\hline
\end{tabular}

During real-time operation, Puma 761 is supplied with the recorded vectors of the previous generated path. The tool tip begins from an initial location and then follows the generated path. The results for two planes are illustrated in Figures $9 \mathrm{~b}$ and $9 \mathrm{c}$ while in Figure $9 \mathrm{~d}$ the graph of angles with respect to the recorded samples (one sample/cycle) is illustrated.

\subsection{Discussion}

The above experimental results show the good quality of the generated path. It can be improved by reducing cycle time. This is possible by executing application HumanPT to faster computers, by using a camera with higher frame rate and 


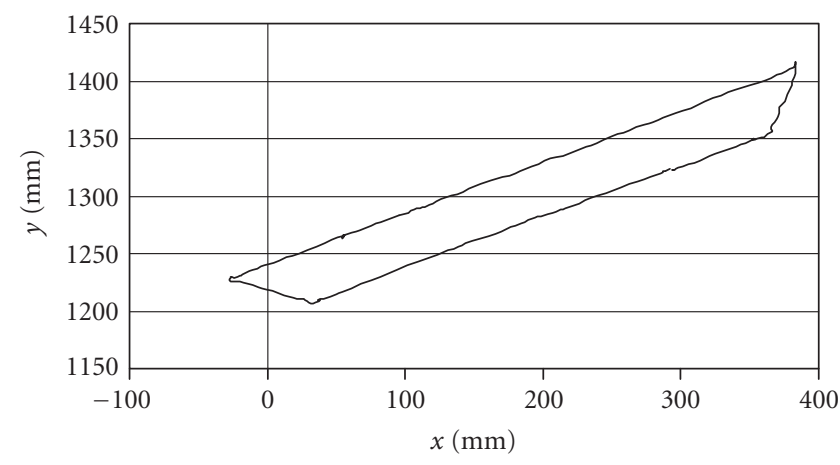

(a)

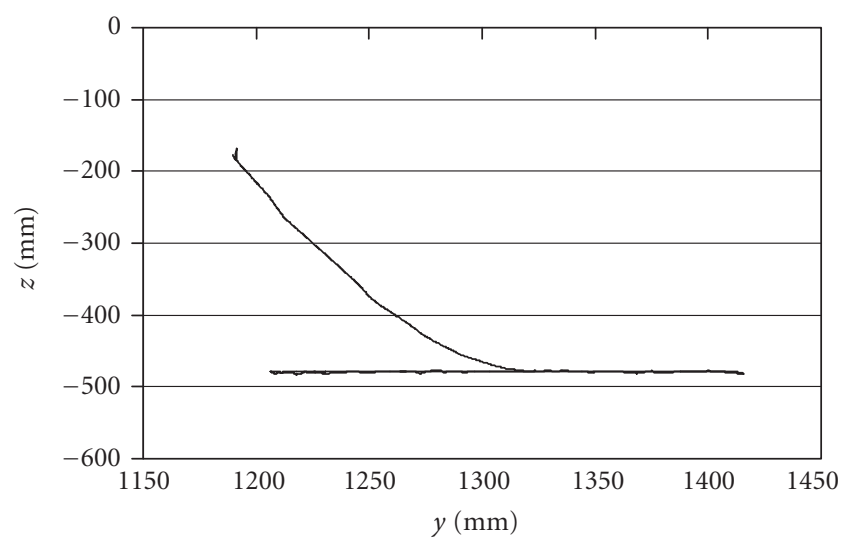

(c)

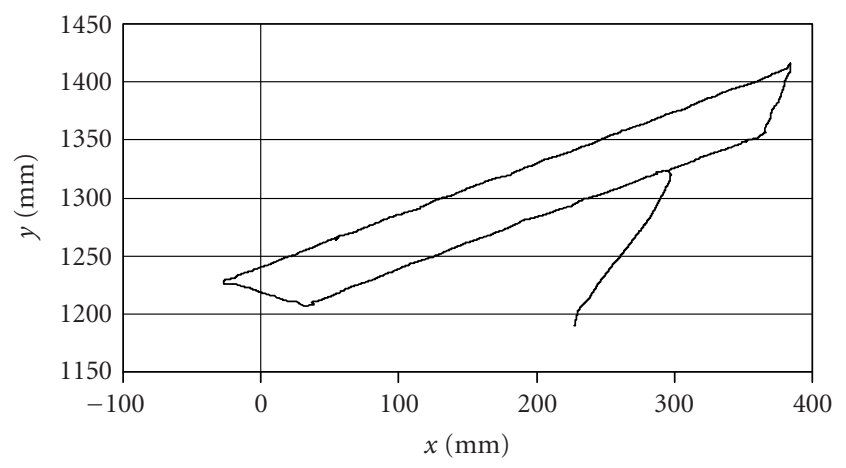

(b)

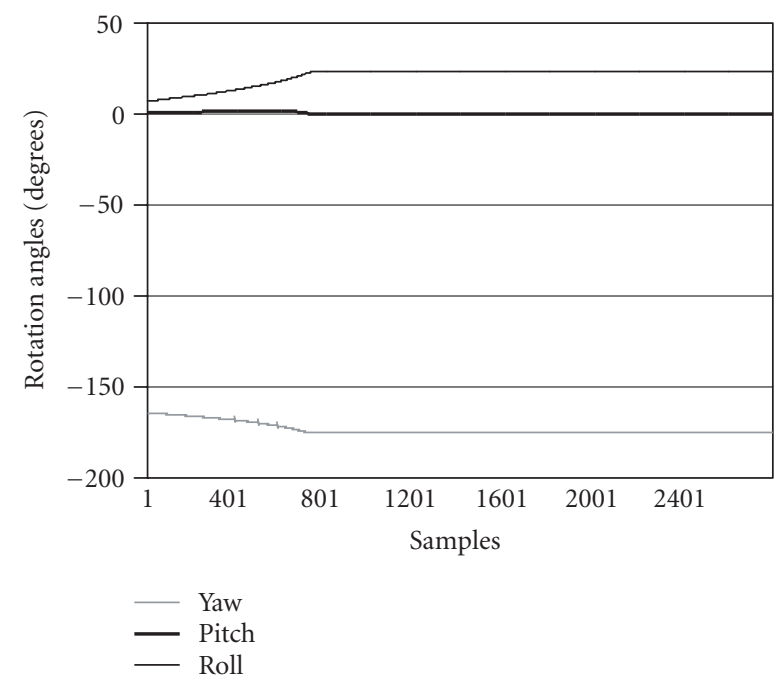

(d)

FIGURE 9: (a) Path generated by means of a trapezium-shaped aluminum piece. Real-time implementation: (b) graph $y$ versus $x$, (c) graph $z$ versus $y$, and (d) graph of rotation angles with respect to recorded samples. Coordinates are considered with respect to the world frame.

TABLE 3: Errors as PSVS tracks the TOB following a trapezium-shaped path (mm).

\begin{tabular}{l|cccccc}
\hline Error & $x_{W G}$ & $y_{W G}$ & $z_{W G}$ & $\psi_{W G}$ & $\theta_{W G}$ \\
\hline ME & 0.1376 & -0.428 & $-4 \mathrm{E}-04$ & 0.0007 & $-7 \mathrm{E}-04$ & 0.0008 \\
MAE & 0.9949 & 0.7873 & 0.392 & 0.822 & 0.8333 \\
MaxE & 3.934 & 3.8898 & 1.344 & 3.181 & 2.588 \\
\hline
\end{tabular}

by selecting the proper metal driver. The control loop is robust and light sources remain in the field of view of PSVS, in an almost steady position, if TOB velocities are smaller than robot velocities. In any other case, for safety reasons, the robot remains in its last state or it returns to an initial prespecified state (Figure 5, Stop on Current Location check box). Moreover the software developed examines cases where only one or two light sources are found in the field of view of PSVS. Then, only position control is possible. However, current TOB state can accurately be recorded if PSVS is carefully calibrated.

\section{CONCLUSIONS}

In this paper an integrated system for robot path generation for a welding system was described. A part of the robotic software application called HumanPT permits the visual servo control of the robot by means of the TOB, PSVS, and the control scheme proposed. At the same time the computed poses of the TOB or of the driver attached to the TOB are recorded. The communication and control of the robot are made by means of two personal computers through ALTER port working at 38400 bps. Despite inaccuracies of the Puma 


\begin{tabular}{|c|c|c|c|c|c|c|c|c|}
\hline$X$ & $X$ & $X$ & $X$ & $C$ & $X$ & $X$ & $X$ & $X$ \\
\hline$X$ & $X$ & $X$ & $X$ & $C$ & $X$ & $X$ & $X$ & $X$ \\
\hline$X$ & $X$ & $X$ & $X$ & $C$ & $X$ & $X$ & $X$ & $X$ \\
\hline$X$ & $X$ & $X$ & $X$ & $C$ & $X$ & $X$ & $X$ & $X$ \\
\hline$C$ & $C$ & $C$ & $C$ & $C$ & $C$ & $C$ & $C$ & $C$ \\
\hline$X$ & $X$ & $X$ & $X$ & $C$ & $X$ & $X$ & $X$ & $X$ \\
\hline$X$ & $X$ & $X$ & $X$ & $C$ & $X$ & $X$ & $X$ & $X$ \\
\hline$X$ & $X$ & $X$ & $X$ & $C$ & $X$ & $X$ & $X$ & $X$ \\
\hline$X$ & $X$ & $X$ & $X$ & $C$ & $X$ & $X$ & $X$ & $X$ \\
\hline
\end{tabular}

(a)

\begin{tabular}{|l|l|l|l|l|l|l|l|l|l|l|}
\hline$S$ & $X$ & $X$ & $X$ & $X$ & $X$ & $X$ & $X$ & $X$ & $X$ & $S$ \\
\hline$X$ & $S$ & $X$ & $X$ & $X$ & $X$ & $X$ & $X$ & $X$ & $S$ & $X$ \\
\hline$X$ & $X$ & $S$ & $X$ & $X$ & $X$ & $X$ & $X$ & $S$ & $X$ & $X$ \\
\hline$X$ & $X$ & $X$ & $S$ & $X$ & $X$ & $X$ & $S$ & $X$ & $X$ & $X$ \\
\hline$X$ & $X$ & $X$ & $X$ & $S$ & $X$ & $S$ & $X$ & $X$ & $X$ & $X$ \\
\hline$X$ & $X$ & $X$ & $X$ & $X$ & $S$ & $X$ & $X$ & $X$ & $X$ & $X$ \\
\hline$X$ & $X$ & $X$ & $X$ & $S$ & $X$ & $C$ & $X$ & $X$ & $X$ & $X$ \\
\hline$X$ & $X$ & $X$ & $S$ & $X$ & $X$ & $X$ & $C$ & $X$ & $X$ & $X$ \\
\hline$X$ & $X$ & $S$ & $X$ & $X$ & $X$ & $X$ & $X$ & $C$ & $X$ & $X$ \\
\hline$X$ & $S$ & $X$ & $X$ & $X$ & $X$ & $X$ & $X$ & $X$ & $C$ & $X$ \\
\hline$S$ & $X$ & $X$ & $X$ & $X$ & $X$ & $X$ & $X$ & $X$ & $X$ & $C$ \\
\hline
\end{tabular}

(c) (b)

\begin{tabular}{|l|l|l|l|l|l|l|l|l|l|l|l|l|l|}
\hline$S$ & $X$ & $X$ & $X$ & $X$ & $X$ & $X$ & $X$ & $X$ & $X$ & $S$ \\
\hline$X$ & $S$ & $X$ & $X$ & $X$ & $X$ & $X$ & $X$ & $X$ & $S$ & $X$ \\
\hline$X$ & $X$ & $S$ & $X$ & $X$ & $X$ & $X$ & $X$ & $S$ & $X$ & $X$ \\
\hline$X$ & $X$ & $X$ & $S$ & $X$ & $X$ & $X$ & $S$ & $X$ & $X$ & $X$ \\
\hline$X$ & $X$ & $X$ & $X$ & $S$ & $X$ & $S$ & $X$ & $X$ & $X$ & $X$ \\
\hline$X$ & $X$ & $X$ & $X$ & $X$ & $C$ & $X$ & $X$ & $X$ & $X$ & $X$ \\
\hline$X$ & $X$ & $C$ & $X$ & $X$ \\
\hline$X$ & $X$ & $C$ & $X$ & $X$ \\
\hline$C$ & $C$ & $C$ & $C$ & $C$ \\
\hline$X$ & $X$ & $C$ & $X$ & $X$ \\
\hline$X$ & $X$ & $C$ & $X$ & $X$ \\
\hline$X$ & $X$ & $X$ & $X$ & $S$ & $X$ & $C$ & $X$ & $X$ & $X$ & $X$ \\
\hline$X$ & $X$ & $X$ & $S$ & $X$ & $X$ & $X$ & $C$ & $X$ & $X$ & $X$ \\
\hline$X$ & $S$ & $X$ & $X$ & $X$ & $X$ & $X$ & $X$ & $X$ & $C$ & $X$ \\
\hline$S$ & $X$ & $X$ & $X$ & $X$ & $X$ & $X$ & $X$ & $X$ & $X$ & $C$ \\
\hline
\end{tabular}

$\mid$\begin{tabular}{|c|c|c|c|c|c|c|c|c|c|c|c|c|c|}
\hline$S$ & $X$ & $X$ & $X$ & $X$ & $X$ & $X$ & $X$ & $X$ & $X$ & $S$ \\
\hline$X$ & $S$ & $X$ & $X$ & $X$ & $X$ & $X$ & $X$ & $X$ & $S$ & $X$ \\
\hline$X$ & $X$ & $S$ & $X$ & $X$ & $X$ & $X$ & $X$ & $S$ & $X$ & $X$ \\
\hline$X$ & $X$ & $X$ & $S$ & $X$ & $X$ & $X$ & $S$ & $X$ & $X$ & $X$ \\
\hline$X$ & $X$ & $X$ & $X$ & $S$ & $X$ & $S$ & $X$ & $X$ & $X$ & $X$ \\
\hline$X$ & $X$ & $C$ & $X$ & $X$ \\
\hline$C$ & $C$ & $C$ & $C$ & $C$ \\
\hline$X$ & $X$ & $C$ & $X$ & $X$ \\
\hline$X$ & $X$ & $C$ & $X$ & $X$ \\
\hline$X$ & $X$ & $X$ & $X$ & $X$ & $C$ & $X$ & $X$ & $X$ & $X$ & $X$ \\
\hline$X$ & $X$ & $X$ & $X$ & $S$ & $X$ & $C$ & $X$ & $X$ & $X$ & $X$ \\
\hline$X$ & $X$ & $X$ & $S$ & $X$ & $X$ & $X$ & $C$ & $X$ & $X$ & $X$ \\
\hline$X$ & $X$ & $S$ & $X$ & $X$ & $X$ & $X$ & $X$ & $C$ & $X$ & $X$ \\
\hline$X$ & $S$ & $X$ & $X$ & $X$ & $X$ & $X$ & $X$ & $X$ & $C$ & $X$ \\
\hline$S$ & $X$ & $X$ & $X$ & $X$ & $X$ & $X$ & $X$ & $X$ & $X$ & $C$ \\
\hline
\end{tabular}

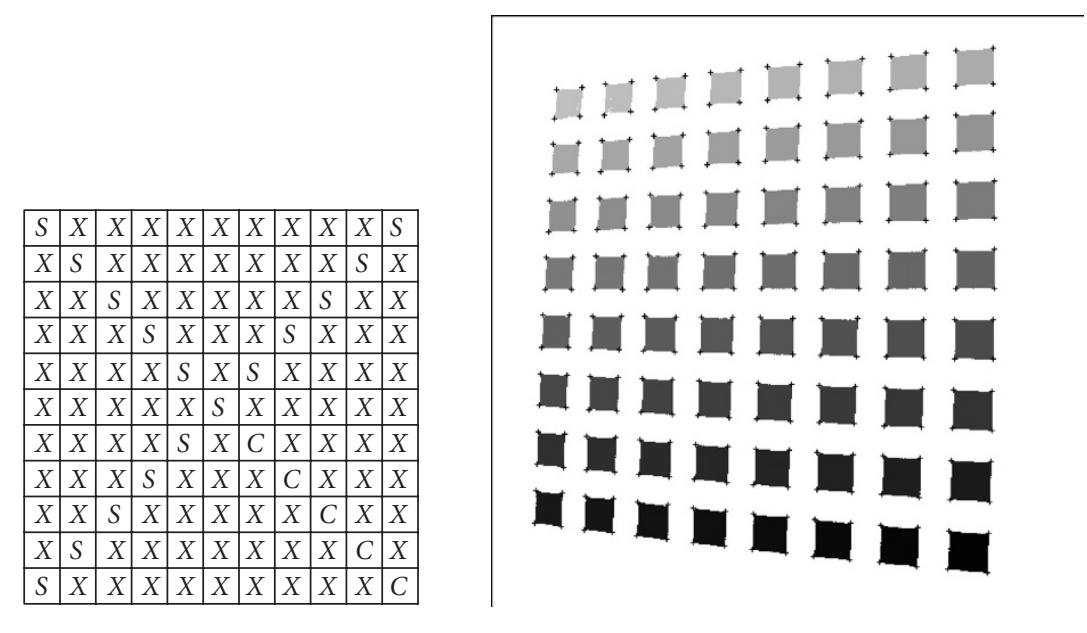

(d)

Figure 10: Corner detection algorithm. (a) Cross-shape templates, (b) $X$-shape template for corner detection, “C” type, (c) $X$-shape template for corner detection, " $S$ " type, and (d) the ordered segmentation of squares and corners detected are illustrated.

robot and reported problems of ALTER port [38], the pseudo stereo visual servo loop, as presented, ensures the robustness of the system and the recently developed PSVS the accuracy of measurements. The proposed method can be applied in manipulators or in mobile robots to generate any path just following the moving TOB. Drivers attached to the TOB may be replaced according to the requirements of each application.

\section{APPENDIX}

The corner detection algorithm proposed in [41] was developed to support Zhang calibration method [40]. It finds corners in a pattern with squares by means of templates even in cases where squares in images have big slope or images are distorted due to camera lens. While, in general, it is considered that template-based methods are slower than geometrybased algorithms; the algorithm is fast enough because a small number of pixels of the proposed templates are used.

Two basic concepts are used. The first one concerns the method of separating each square of the binary image. For this reason the concept of seed is used, that is, a pixel with a unique specified color or gray scale value. In patterns, in which shapes can be considered as separated entities, in each shape (i.e., a square) a seed is planted. Then, this seed can be propagated only to this entity. In this way, the segmentation of an image is possible and, if it is desired, with a specified order of the created segments. The last idea is implemented in the proposed algorithm. The segmentation order is specified using as features for the estimation of the new seed the center and the slope of the previous segmented square. Two criteria for the selection of a seed are used. The first is its distance from a specified initial point. The second criterion is the following: the candidate pixel must be the center pixel of a $9 \times 9$ or $5 \times 5$ template (the template used depends on the case) with cross-shape (Figure 10a). The last criterion is used to make the pixel selection insensitive to noise.

The second concept concerns the method used for corner detection. Here, only two $11 \times 11$ templates (Figures $10 \mathrm{~b}$, 10c) are used. In these templates a small number of pixels are convoluted with the image pixels. The scanning area is also limited to a region around the current square with the seed. This way the algorithm is quite fast. The orientation of the templates depends on the orientation of the searching corner. In Figures 10b, 10c, " $S$ " represents pixels with the substrate color, " $C$ " pixels with the square color, and " $X$ " are 
"don't care" pixels. In the first template (Figure 10b) the center pixel is type " $C$ " and in the second template (Figure 10c) the center pixel is type " $S$."

As it is easily realized from these figures the templates are " $X$ " shape where one leg is colored with the square color and the other three legs are colored with the substrate color. The detecting pixel is the center pixel of the templates. Rotating these two basic templates $90^{\circ}$ each time and then applying them to the image, the detection of all corner pixels is possible. The interesting corner point is the peak formed around corner area from the change of the substrate color to the square color and vice versa. This peak may be the mean value of two pixels in a well-formed square in a binary image or the mean value of many detected pixels, creating a cluster of pixels around the corner, using the above templates in a binary, gray scale, or color image. Using these slightly different types of templates the inner and the outer side pixels of the corner are simultaneously detected. The result of ordered segmentation and corner detection is depicted in Figure 10d. A complete algorithm for corner detection, as well as calibration facilities by means of the proper graphical user interfaces (parts of HumanPT application), is also provided.

\section{REFERENCES}

[1] J. T. Feddema and O. R. Mitchell, "Vision-guided servoing with feature-based trajectory generation," IEEE Trans. Robot. Automat., vol. 5, no. 5, pp. 691-700, 1989.

[2] Y. Kuniyoshi, M. Inaba, and H. Inoue, "Learning by watching: extracting reusable task knowledge from visual information of human performance," IEEE Trans. Robot. Automat., vol. 10, no. 6, pp. 799-822, 1994.

[3] A. Ude and R. Dillmann, "Trajectory reconstruction from stereo image sequences for teaching robot paths," in Proc. 24th International Symposium on Industrial Robots (ISIR '93), pp. 407-414, Tokyo, Japan, November 1993.

[4] A. Ude and R. Dillmann, "Vision-based robot path planning," in Advances in Robot Kinematics and Computational Geometry, pp. 505-512, Kluwer Academic Publishers, Dordrecht, The Netherlands, 1994.

[5] A. Ude and R. Dillmann, "Robot motion specification: a vision-based approach," Surveys on Mathematics for Industry, vol. 5, no. 2, pp. 109-131, 1995.

[6] S. Kang, Robot instruction by human demonstration, Ph.D. thesis, Robotics Institute, Carnegie Mellon University, Pittsburgh, Pa, USA, 1994.

[7] B. Brunner, K. Arbter, G. Hirxinger, and R. Koeppe, "Programming robots via learning by showing in a virtual environment," in Proc. Virtual Reality World '95, IDG Conferences and Seminars, pp. 63-72, Munich, Germany, 1995.

[8] R. Voyles and P. K. Khosla, "Gesture-based programming, part I: a multi-agent approach," in Proc. ANNIE: Artificial Neural Networks, Fuzzy Logic and Evolutionary Programming for Designing Smart Engineering Systems, pp. 299-304, ASME Press, St. Louis, Mo, USA, November 1996.

[9] C. G. Johnson and D. Marsh, "Modeling robot manipulators in a CAD environment using B-splines," in Proc. IEEE International Joint Symposia on Intelligence and Systems (IJSIS '96), $\mathrm{N}$. G. Bourbakis, Ed., pp. 194-201, Rockville, Md, USA, November 1996.

[10] P. N. Sabes, The planning of visually guided arm movements: feedback perturbation and obstacle avoidance studies, Ph.D. thesis, MIT, Cambridge, Mass, USA, 1996.
[11] L. F. Gu, "Visual guidance of robot motion," M.S. thesis, University of Western Australia, Crawley, Western Australia, 1996.

[12] M. Päschke and J. Pauli, "Vision based learning of gripper trajectories for a robot arm," in Proc. International Symposium on Automotive Technology and Automation, pp. 235-242, Florence, Italy, 1997.

[13] R. Sharma and H. Sutanto, "A framework for robot motion planning with sensor constraints," IEEE Trans. Robot. Automat., vol. 13, no. 1, pp. 61-73, 1997.

[14] E. Berry, P. Martinet, and J. Gallice, "Trajectory generation by visual servoing," in Proc. IEEE/RSJ International Conference on Intelligent Robots and Systems (IROS '97), vol. 2, pp. 10661072, Grenoble, France, September 1997.

[15] A. Ruf and R. Horaud, "Visual trajectories from uncalibrated images," in Proc. Workshop on New Trends in Image-Based Robot Servoing, Proc. IEEE/RSJ International Conference On Intelligent Robots and Systems, pp. 83-91, Grenoble, France, September 1997.

[16] P. Kim, S. Rhee, and C. H. Lee, "Automatic teaching of welding robot for free-formed seam using laser vision sensor," $O p$ tics and Lasers in Engineering, vol. 31, no. 3, pp. 173-182, 1999.

[17] X. F. Zha and H. Du, "Generation and simulation of robot trajectories in a virtual CAD-based off-line programming environment," International Journal of Advanced Manufacturing Technology, vol. 17, no. 8, pp. 610-624, 2001.

[18] Y. Mezouar and F. Chaumette, "Path planning for robust image-based control," IEEE Trans. Robot. Automat., vol. 18, no. 4, pp. 534-549, 2002.

[19] T. Pachidis, J. Lygouras, and P. Tsalidis, "A graphical user interface for the initial path generation of a robotic manipulator for an arc welding system," in Proc. 2nd WSEAS International Conference on Robotics, Distance Learning and Intelligent Communication Systems (ICRODIC '02), pp. 1601-1607, Skiathos Island, Greece, September 2002.

[20] B. Espiau, F. Chaumette, and P. Rives, "A new approach to visual servoing in robotics," IEEE Trans. Robot. Automat., vol. 8, no. 3, pp. 313-326, 1992.

[21] N. P. Papanikolopoulos, P. K. Khosla, and T. Kanade, "Visual tracking of a moving target by a camera mounted on a robot: a combination of control and vision," IEEE Trans. Robot. Automat., vol. 9, no. 1, pp. 14-35, 1993.

[22] W. Wilson, C. Hulls, and G. Bell, "Relative end-effector control using cartesian position-based visual servoing," IEEE Trans. Robot. Automat., vol. 12, no. 5, pp. 684-696, 1996.

[23] P. Martinet, "Comparison of visual servoing techniques: experimental results," in Proc. European Control Conference (ECC '99), pp. 1059-1064, Karlsruhe, Germany, AugustSeptember 1999.

[24] E. Cervera and P. Martinet, "Visual servoing with indirect image control and a predictable camera trajectory," in Proc. IEEE/RSJ International Conference on Intelligent Robots and Systems (IROS '99), vol. 1, pp. 381-386, Kyongju, South Korea, October 1999.

[25] J. A. Gangloff and M. F. de Mathelin, "Visual servoing of a 6DOF manipulator for unknown 3-D profile following," IEEE Trans. Robot. Automat., vol. 18, no. 4, pp. 511-520, 2002.

[26] R. Mahony, T. Hamel, and F. Chaumette, "A decoupled image space approach to visual servo control of a robotic manipulator," in Proc. IEEE International Conference on Robotics and Automation (ICRA '02), vol. 4, pp. 3781-3786, Washington, DC, USA, May 2002.

[27] E. Cervera, F. Berry, and P. Martinet, "Is 3D useful in stereo visual control?" in Proc. IEEE International Conference on Robotics and Automation (ICRA '02), vol. 2, pp. 1630-1635, Washington, DC, USA, May 2002. 
[28] B. Thuilot, P. Martinet, L. Cordesses, and J. Gallice, "Position based visual servoing: keeping the object in the field of vision," in Proc. IEEE International Conference on Robotics and Automation (ICRA '02), vol. 2, pp. 1624-1629, Washington, DC, USA, May 2002.

[29] E. Malis, "Vision-based control invariant to camera intrinsic parameters: stability analysis and path tracking," in Proc. IEEE International Conference on Robotics and Automation (ICRA '02), vol. 1, pp. 217-222, Washington, DC, USA, May 2002.

[30] G. Hager, "A modular system for robust positioning using feedback from stereo vision," IEEE Trans. Robot. Automat., vol. 13 , no. 4, pp. 582-595, 1997.

[31] B. Lamiroy, B. Espiau, N. Ahdreff, and R. Horaud, "Controlling robots with two cameras: how to do it properly," in Proc. IEEE International Conference on Robotics and Automation (ICRA '00), pp. 2100-2105, San Fransisco, Calif, USA, April 2000.

[32] E. Malis, F. Chaumette, and S. Boudet, "Multi-cameras visual servoing," in Proc. IEEE International Conference on Robotics and Automation (ICRA '00), vol. 4, pp. 3183-3188, San Francisco, Calif, USA, April 2000.

[33] E. Cervera, F. Berry, and P. Martinet, "Stereo visual servoing with a single point: a comparative study," in Proc. 9th IEEE International Conference on Advanced Robotics (ICAR '01), pp. 213-218, Budapest, Hungary, August 2001.

[34] P. Martinet and E. Cervera, "Stacking jacobians properly in stereo visual servoing," in Proc. IEEE International Conference on Robotics and Automation (ICRA '01), pp. 717-722, Seoul, Korea, May 2001.

[35] P. Martinet and E. Cervera, "Image-based stereo visual servoing: 2D vs 3D features," in Proc. IEEE International Conference on Robotics and Automation (ICRA '02), vol. 2, pp. 1630-1635, Washington, DC, USA, May 2002.

[36] S. Hutchinson, G. Hager, and P. Corke, "A tutorial in visual servo control," IEEE Trans. Robot. Automat., vol. 12, no. 5, pp. 651-670, 1996.

[37] T. Pachidis and J. Lygouras, "A pseudo stereo vision system as a sensor for real time path control of a robot," in Proc. 19th IEEE Instrumentation and Measurement Technology Conference (IMTC '02), vol. 2, pp. 1589-1594, Anchorage, Alaska, USA, May 2002.

[38] G. Hager, S. Hutchinson, and P. Corke, "Tutorial TT3: a tutorial on visual servo control," in conjunction with IEEE Conference on Robotics and Automation, Minneapolis, Minn, USA, April 1996.

[39] T. Pachidis and J. Lygouras, "Pseudo stereo vision system: modifications for accurate measurements in 3-D space using camera calibration," in Proc. ISA/IEEE Sensors for Industry Conference, pp. 66-70, Houston, Tex, USA, November 2002.

[40] Z. Zhang, "A flexible new technique for camera calibration," IEEE Trans. Pattern Anal. Machine Intell., vol. 22, no. 11, pp. 1330-1334, 2000.

[41] T. Pachidis, J. Lygouras, and V. Petridis, "A novel corner detection algorithm for camera calibration and calibration facilities," in Proc. 2nd WSEAS International Conference on Signal Processing and Computational Geometry and Vision, pp. 69116916, Rethymno, Greece, July 2002.

[42] H. Zhuang, Z. S. Roth, and R. Sudhakar, "Simultaneous robot/world and tool/flange calibration by solving homogeneous transformation equations of the form $\mathrm{AX}=\mathrm{YB}$," IEEE Trans. Robot. Automat., vol. 10, no. 4, pp. 549-554, 1994.

[43] S. G. Tzafestas, Robotic Systems: Advanced Techniques and Applications, Kluwer Academic Publishers, Boston, Mass, USA, 1992.
Theodore P. Pachidis was born in Drama, Greece, in August 1962. He received the B.S. degree in physics and the M.S. degree in electronics from the Aristotle University of Thessaloniki in 1985 and 1989, respectively. Since 1989 he has been a Teacher of physics and electronics. He is currently working toward his Ph.D. degree in robotics and machine vision systems in the Department of Electrical and Computer Engineer-

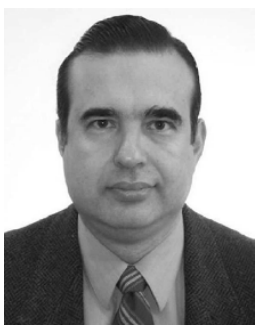

ing, Democritus University of Thrace. From 1996 to 1998, he was a Schoolmaster of the Public Institute of Professional Training at Kavala. His interests include the design and construction of electronic devices and systems, robotics, machine vision systems, visual $\mathrm{C}++$, and microcontroller programming. He is a Member of the IEEE and the IEEE Computer Society.

Kostas N. Tarchanidis received the Diploma degree in electrical engineering from the Democritus University of Thrace and his Ph.D. degree in robotics from the Department of Electrical Engineering and Electronics, University of Liverpool. He is presently an Associate Professor in the Department of Petroleum Technology, Technological Educational Institute of Kavala, Kavala, Greece. He is Dean of the School of Engineering. He is a Member of the Technical Chamber of Greece and the IEEE.

John N. Lygouras was born in Kozani, Greece, in May 1955. He received the Diploma and the Ph.D. degrees in electrical engineering from the Democritus University of Thrace, Greece, in 1982 and 1990, respectively, both with honors. From 1982 he was a Research Assistant and since 2000 he has been an Associate Professor in the Department of Electrical and Computer Engineering, Democritus University of Thrace.

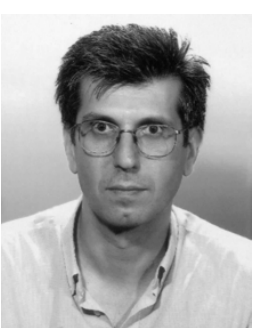
In 1997 he spent six months in the Department of Electrical Engineering and Electronics, the University of Liverpool, as a Honorary Senior Research Fellow. His research interests are in the field of robotic manipulators trajectory planning and execution. His interests also include the research on analog and digital electronic systems implementation and position control of underwater remotely operated vehicles.

Philippos G. Tsalides was born in Mirina Limnou, Greece, on October 14th, 1953. He received the Diploma degree in electronic engineering from the University of Padova, Italy, in 1979, and the Ph.D. degree in electrical engineering from the Democritus University of Thrace, Greece, in 1985 . He is a Professor of applied electronics in the Department of Electrical and Computer Engineering, Democritus University of Thrace.

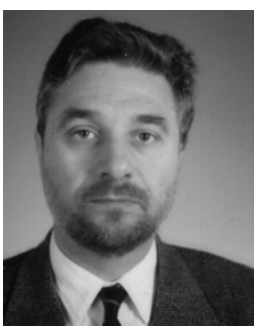
His current research interests include VLSI architectures, VLSI systems, BIST techniques, LANs, WANs, applications of cellular automata in image processing, as well as in computational systems. He has published a number of papers and three textbooks on VLSI systems, on microprocessors, and on automated electronic measurements. 